Received: 13.11 .2017

Revised: 25.12 .2017

Accepted: 26.01 .2018

DOI: $10.17804 / 2410-9908.2018 .1 .034-040$

\title{
ON THE POSSIBILITY OF INCREASING THE SERVICE LIFE OF HIGH-POWER LASER OPTICS THROUGH THE USE OF POLYCRYSTALLINE DIAMOND WINDOWS WITH A CENTRAL MONOCRYSTALLINE AREA
}

\author{
M. V. Rogozhin ${ }^{1, \text { a) }}$, V. E. Rogalin 2,3, b) , M. I. Krymsky ${ }^{1,2}$, I. A. Kaplunov ${ }^{3, \text { c }^{*}}$ \\ ${ }^{1}$ Moscow Institute of Physics and Technology (State University), 9 Institutsky Lane, Dolgoprudny, \\ Moscow Region, 141701, Russian Federation \\ ${ }^{2}$ National Center of Laser Systems and Complexes JSC, \\ 95 Volokolamskoe Hw., Moscow, 125424, Russian Federation \\ ${ }^{3}$ Tver State University, \\ 33 Zhelyabova St. Tver, 170100, Russian Federation \\ a) (iD https://orcid.org/0000-0002-4886-7864, 国 max.salavat@ mail.ru;

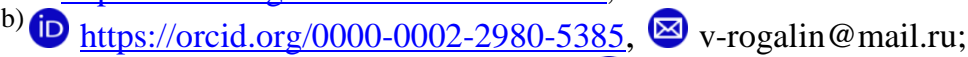 \\ c) (iD https://orcid.org/0000-0002-1726-3451, 0 Kaplunov.IA@tversu.rul.ru \\ *Corresponding author. E-mail: Kaplunov.IA@tversu.rul.ru \\ Address for correspondence: ul. Zhelyabova, 33, 170100, Tver, Russian Federation \\ Tel.: +7 48223228 39; fax: +7 4822322839
}

For high-power $\mathrm{CO}_{2}$ lasers operating at $10-\mu \mathrm{m}$ wavelength there is a serious deficiency of transparent materials. Only expensive semiconductor and dielectric materials with poor mechanical and thermal characteristics are transparent in this spectral region. The construction of an output high-power laser window made of polycrystalline diamond with a single-crystalline central region is considered. Numerical modeling of an optical damage threshold is performed. The results are compared with those for a conventionally designed polycrystalline diamond window. An increase in maximum allowed output radiation power due to the use of a composite window is demonstrated.

Keywords: high-power $\mathrm{CO}_{2}$ laser, output window, polycrystalline diamond, single-crystal diamond, optical damage.

\section{References}

1. Rogalin V.E. Transparent materials for high-power pulsed $\mathrm{CO}_{2}$-lasers // Izvestiya VUZov. Materialy elektronnoy tekhniki, 2013, no. 2, pp. 11-18. (In Russian).

2. Rogalin V.E., Aranchiy S.A. Polycrystalline diamonds - new prospects for power optics and electronics. Integral, 2012, no. 5 (67), pp. 7-9. (In Russian).

3. Rogalin V.E., Ashkenazi E.E., Popovich A.F., Ral'chenko V.G., Konov V.I., Aranchii S.M., Ruzin M.V., Uspenskii S.A. Resistance of diamond optics to high-power fiber laser radiation. Russian Microelectronics, 2012, vol. 41, no. 8, pp. 464-468. DOI: 10.1134/S106373971208015X

4. Kaminskii A.A., Hemley R.J., Lai J., Yan C.S., Mao H.K., Ralchenko V.G.,. Eichler H.J, Rhee H. High-order stimulated Raman scattering in CVD single crystal diamond. Laser Phys. Lett., 2007, vol. 4, iss. 5, pp. 350-353. DOI: 10.1002/lapl.200610127

5. $\quad$ Friel. I., Geoghegan S.L., Twitchen D.J., Scarsbrook G.A. Development of high quality single crystal diamond for novel laser applications. In: Proc. SPIE 7838: Optics and Photonics for Counterterrorism and Crime Fighting VI and Optical Materials in Defense Systems Technology VII, 2010, 783819. 
6. Webster S., Chen Y., Turri G., Bennett A., Wickham B., Bass M. Intrinsic and extrinsic absorption of chemical vapor deposition single-crystal diamond from the middle ultraviolet to the far infrared. J. Opt. Soc. Am. B., 2015, vol. 32, no. 3, pp. 479-484. DOI: 10.1364/JOSAB.32.000479 7. Anoikin E., Muhr A., Bennett An., Twitchen D. J., H. de Wit Diamond optical components for high - power and high-energy laser applications. In: Proc. of SPIE, vol. 9346, 93460T, pp. 1-9. DOI: $10.1117 / 12.2079714$

8. Bogachev A.V., Garanin S.G., Dudov A.M., Eroshenko V.A., Kulikov S.M., Mikaelyan G.T., Panarin V.A., Pautov V.O., Rus A.V., Sukharev S.A. Diode-pumped caesium vapour laser with closed-cycle laser-active medium circulation. Quantum Electronics, 2012, vol. 42, no. 2, pp. 95-98. DOI: 10.1070/QE2012v042n02ABEH014734

9. Ho S., Yan C.S., Liu Z., Mao H.K., Hemley R.J. Prospects for large single crystal CVD diamond. Industrial Diamond Review, 2006, vol. 66, pp. 28-32.

10. Yamada H., Chayahara A., Mokuno Y., Umezawa H., Shikata S., Fujimori N. Fabrication of 1 Inch Mosaic Crystal Diamond Wafers. Applied Physics Express, 2010, vol. 3, no. 5, pp. 051301. DOI: 10.1143/APEX.3.051301

11. Vikharev A.L., Gorbachev A.M., Dukhnovsky M.P., Muchnikov A.B., Ratnikova A.K., Fedorov Yu.Yu. Combined Single-crystalline and Polycrystalline CVD Diamond Substrates for Diamond Electronics. Semiconductors, 2012, vol. 46, no. 2, pp. 263-266. DOI: 10.1134/S1063782612020248

12. Rogozhin M.V., Krimsky M.I., Rogalin V.E., Filin S.A. Modeling the thermomechanical processes in the output window of a high-power CO2 laser. Bulletin of the Russian Academy of Sciences: Physics, 2016, vol. 80, no. 10, pp. 1260-1266. DOI: 10.3103/S1062873816100166

13. Rogozhin M.V., Rogalin V.E. and Krymskii M.I. Thermooptical processes in the window of a high-power gas laser. Optics and Spectroscopy, 2017, vol. 122, no. 5, pp. 843-849. DOI: $10.1134 / \mathrm{S} 0030400 X 17050186$

14. Rogozhin M.V., Rogalin V.E., Krymskij M.I., Filin S.A., Rogalina N.A. High-power laser. RF Patent 2608309. (In Russian). 
Подана в журнал: 13.11 .2017

УДК 67.017

DOI: $10.17804 / 2410-9908.2018 .1 .034-040$

\title{
О ВОЗМОЖНОСТИ ПОВЫШЕНИЯ РЕСУРСА ОПТИКИ ВЫСОКОМОЩНЫХ ЛАЗЕРОВ ЗА СЧЕТ ИСПОЛЬЗОВАНИЯ ПОЛИКРИСТАЛЛИЧЕСКИХ АЛМАЗНЫХ ОКОН С ЦЕНТРАЛЬНОЙ МОНОКРИСТАЛЛИЧЕСКОЙ ОБЛАСТЬЮ
}

\author{
М. В. Рогожин ${ }^{1, \text { a) }}$, В. Е. Рогалин ${ }^{2,3, \text { б) }}$, М. И. Крымский ${ }^{1,2}$, И. А. Каплунов ${ }^{3, \text { в)* }}$ \\ ${ }^{1}$ Московский физико-технический институт \\ 141701, Институтский переулок, д. 9, Долгопрудный, Российская Федерация \\ ${ }^{2}$ АО «Национальный центр лазерных систем и комплексов «Астрофизика» \\ 125424, Волоколамское ш., 95, Москва, Российская Федераџия \\ ${ }^{3}$ Тверской государственный университет \\ 170100, ул. Желябова, 33, Тверь, Российская Федерация \\ a) (iD https://orcid.org/0000-0002-4886-7864, 龱 max.salavat@ mail.ru; \\ б) (iD https://orcid.org/0000-0002-2980-5385, 0 v-rogalin@mail.ru; \\ в) (iD https://orcid.org/0000-0002-1726-3451, 図 Kaplunov.IA@ @ tversu.rul.ru \\ *Ответственный автор. Электронная почта: Kaplunov.IA@tversu.rul.ru \\ Адрес для переписки: ул. Желябова, 33, 170100, г. Тверь, Российская Федерация \\ Тел.: +7 4822 32-28-39; факс: +7 4822 32-28-39
}

Для мощных $\mathrm{CO}_{2}$-лазеров, излучающих в диапазоне 10 мкм, существует острый дефицит прозрачных материалов. В этой области прозрачны лишь некоторые весьма дорогие полупроводниковые и диэлектрические монокристаллы, обладающие низкими механическими и теплофизическими свойствами. Для мощного $\mathrm{CO}_{2}$-лазера рассмотрена конструкция выходного окна, изготовленного из поликристаллического алмаза с центральной областью, выполненной из монокристалла алмаза. Проведено численное моделирование оптической стойкости окна. Результаты сопоставлены с параметрами оптической стойкости окна традиционной конструкции из поликристаллического алмаза. Показано, что использование такого окна позволит вдвое увеличить выходную мощность лазера.

Ключевые слова: мощный $\mathrm{CO}_{2}$-лазер, выходное окно, поликристаллический алмаз, монокристаллический алмаз, оптическая стойкость.

\section{1. Введение}

Окно для вывода излучения - наиболее уязвимый конструктивный элемент мощных газовых лазеров. Например, при осаждении на окно частиц пыли из окружающей среды оно начинает интенсивно поглощать излучение, что приводит к локальному нагреву материала окна. Для мощных $\mathrm{CO}_{2}$-лазеров, излучающих в области 10,6 мкм, проблема вывода излучения является наиболее острой. В диапазоне 10 мкм существует острый дефицит прозрачных материалов [1]. В этой области прозрачны лишь некоторые полупроводниковые и диэлектрические монокристаллы, обладающие низкими механическими и теплофизическими свойствами [1]. Кроме того, эти кристаллы трудоёмки в изготовлении и весьма дороги. Попытки получения этих материалов в виде более дешевых керамик или поликристаллов показали, что в большинстве случаев у них заметно возрастает коэффициент поглощения. Поэтому в мощных лазерах эти материалы (кроме $\mathrm{ZnSe}$ ) не применяются.

Оценки показывают, что в окне мощных $\mathrm{CO}_{2}$-лазеров выделяется в виде тепла 0,1-1\% выходной мощности, т. е. при мощности лазера 10-100 кВт эта величина может достигать 10-1000 Вт [2, 3].

For citation: On the possibility of increasing the service life of high-power laser optics through the use of polycrystalline diamond windows with a central monocrystalline area / M. V. Rogozhin, V .E. Rogalin, M. I. Krymsky, I. A. Kaplunov // Diagnostics, Resource and Mechanics of materials and structures. - 2018. - Iss. 1. - P. 34-40. - DOI: 10.17804/24109908.2018.1.034-040. 
В результате может происходить образование термолинзы, приводящей к увеличению расходимости исходящего излучения, и, возможно, разрушение окна. Поэтому именно выходное окно является конструктивным элементом лазера, ограничивающим его максимально возможную мощность генерируемого излучения и качество генерируемого излучения.

Поэтому проблема изготовления выходных окон и других прозрачных оптических элементов для мощных лазеров, позволяющих выдерживать длительное время лучевые нагрузки мультикиловаттной мощности при одновременном обеспечении минимально возможной расходимости исходящего излучения, в настоящее время встала достаточно остро.

Появление нового конструкционного материала - поликристаллического алмаза, обладающего неплохими оптическими свойствами и уникальными теплофизическими, механическими и химическими свойствами, вызвало весьма успешные эксперименты по применению их в мощных $\mathrm{CO}_{2}$-лазерах [2]. Несмотря на значительную стоимость этого материала, его использование оказалось экономически выгодным, так как позволяет получить результаты, недостижимые с другими материалами.

Поликристаллический алмаз, будучи прозрачным материалом в диапазоне от ультрафиолетовых до сверхвысоких частот (за исключением области фононного поглощения 2-6 мкм) [2], обладает самым высоким коэффициентом теплопроводности из известных материалов - порядка 2000 (Вт/м) х К (для сравнения коэффициент теплопроводности меди $380(\mathrm{BT} / \mathrm{M})$ х К)) и очень низким коэффициентом термического расширения, как у инвара. Причём, теплопроводность алмаза можно увеличить в $~ 1,5$ раза путем использования изотопически чистого углерода для выращивания алмазной пластины, используемой для изготовления окна мощного лазера [4].

Поскольку возможно получение поликристаллических алмазов с коэффициентом поглощения на длине волны 1,06 мкм $\left(0,003-0,03 \mathrm{~cm}^{-1}\right.$ [5-7]), что в несколько раз ниже, чем на длине волны 10,6 мкм, открываются перспективы применения алмазной оптики и для твердотельных лазеров. При эксплуатации технологических непрерывных иттербиевых волоконных лазеров с рабочей длиной волны 1,07 мкм, мощностью несколько десятков киловатт возникла проблема, связанная с частым выходом из строя защитного окна объектива. Та же проблема характерна и для активно развивающихся в настоящее время лазеров на парах щелочных металлов, излучающих в ближней ИК-области [8]. Однако проблемой для использования поликристаллических алмазов в ближней ИК области является рассеяние на границах монокристаллических зёрен, заметно возрастающее с уменьшением длины волны.

Применение алмазных окон во многом обусловлено широким развитием щелевых $\mathrm{CO}_{2}$ лазеров, для которых характерен компактный вывод излучения с высокой плотностью мощности.

Ведущие фирмы - производители $\mathrm{CO}_{2}$-лазеров, например TRUMPF, ROFIN-SINAR, выпускают технологические щелевые $\mathrm{CO}_{2}$-лазеры мощностью 3-8 кВт только с алмазным охлаждаемым окном [2]. Технология получения поликристаллических алмазов в настоящее время развита настолько, что получаемый материал по своим оптико-физическим свойствам близок к монокристаллам высококачественного природного алмаза типа ІІа. Однако монокристаллы, тем не менее, имеют меньший коэффициент поглощения, значительно меньший коэффициент рассеяния (вследствие отсутствия границ зёрен) и более высокий предел прочности [5-7]. К сожалению, стоимость искусственных монокристаллов алмаза крайне резко растёт с увеличением размеров, к тому же их технологически достижимые размеры, как правило, недостаточны для изготовления оптических элементов, реально применяемых в мощных $\mathrm{CO}_{2}$-лазерах. Одним из основных факторов, сдерживающих широкое применение изделий из монокристаллического алмаза, является малый геометрический размер алмазных подложек, на которых происходит эпитаксиальный рост, при получении монокристаллов методом плазмохимического осаждения из газовой фазы в вакууме (CVD-метод). Метод позволяет получать достаточно крупные монокристаллы даже ювелирного качества [9]. В настоящее время монокристаллический алмаз выращивается на подложках (размером в основном до 5-10 мм) из ис-

For citation: On the possibility of increasing the service life of high-power laser optics through the use of polycrystalline diamond windows with a central monocrystalline area / M. V. Rogozhin, V .E. Rogalin, M. I. Krymsky, I. A. Kaplunov // Diagnostics, Resource and Mechanics of materials and structures. - 2018. - Iss. 1. - P. 34-40. - DOI: 10.17804/24109908.2018.1.034-040. 
кусственного алмаза, получаемого в аппаратах высокого давления и высокой температуры. Этот размер, как правило, недостаточен для применения в мощных лазерах. Наибольший

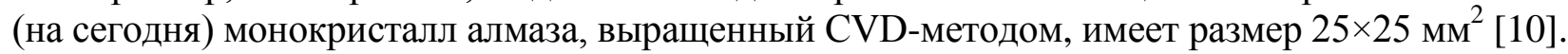

Комбинированные пластины поликристаллического алмаза с монокристаллическими включениями получены в работах [11] и уже успешно применяются в микроэлектронике. Монокристалл используется по основной задаче, а поликристаллическая часть применяется как теплоотводящий элемент.

Цель исследования: разработать конструкцию выходного окна, изготовленного из поликристаллического алмаза с центральной монокристаллической областью; при помощи численного моделирования рассчитать его оптическую стойкость и сравнить ее с оптической стойкостью окна, целиком изготовленного из поликристалла.

\section{2. Постановка задачи и методы решения}

Для моделирования поведения окна под воздействием мощного лазерного излучения была применена математическая модель, ранее описанная в [12-14], скорректированная с учетом двухкомпонентной структуры окна. Монокристаллическая (1) и поликристаллическая (2) части окна считались находящимися в плотном тепловом и механическом контакте, чем обусловлен выбор соответствующих граничных условий в тепловой и механической частях расчетной модели.

$$
\begin{gathered}
Q(r, z)=\left\{\begin{array}{c}
\frac{2 P_{0} \alpha_{1}}{\pi W_{0}^{2}} e^{-\alpha_{1} z} \mathrm{Y} e^{-2 \frac{r^{2}}{W_{0}^{2}}}, 0 \leq r \leq R_{1}, \\
\frac{2 P_{0} \alpha_{2}}{\pi W_{0}^{2}} e^{-\alpha_{2} z} \mathrm{Y} e^{-2 \frac{r^{2}}{W_{0}^{2}}}, R_{1} \leq r \leq R_{2}
\end{array}\right. \\
\left\{\begin{array}{c}
\rho c \frac{\partial T}{\partial t}=\frac{1}{r} \frac{\partial}{\partial r}\left(\lambda_{1} r \frac{\partial T}{\partial r}\right)+\lambda_{1} \frac{\partial^{2} T}{\partial \mathrm{z}^{2}}+Q(r, z), \quad 0 \leq r \leq R_{1}, \\
\rho c \frac{\partial T}{\partial t}=\frac{1}{r} \frac{\partial}{\partial r}\left(\lambda_{2} r \frac{\partial T}{\partial r}\right)+\lambda_{2} \frac{\partial^{2} T}{\partial \mathrm{z}^{2}}+Q(r, z), \quad R_{1} \leq r \leq R_{2}, \\
-\lambda_{1}\left(\frac{\partial T}{\partial r}\right)_{r=R 1-0}=-\lambda_{2}\left(\frac{\partial T}{\partial r}\right)_{r=R 1+0}, \\
T\left(R_{1}-0, z\right)=T\left(R_{1}+0, z\right),
\end{array}\right.
\end{gathered}
$$

где $c, c$ - плотность и удельная теплоемкость алмаза (были приняты одинаковыми для монои поликристалла), $\alpha_{1}$ и $\alpha_{2}, \lambda_{1}$ и $\lambda_{2}-$ коэффициенты поглощения излучения и теплопроводности для моно- и поликристалла алмаза соответственно.

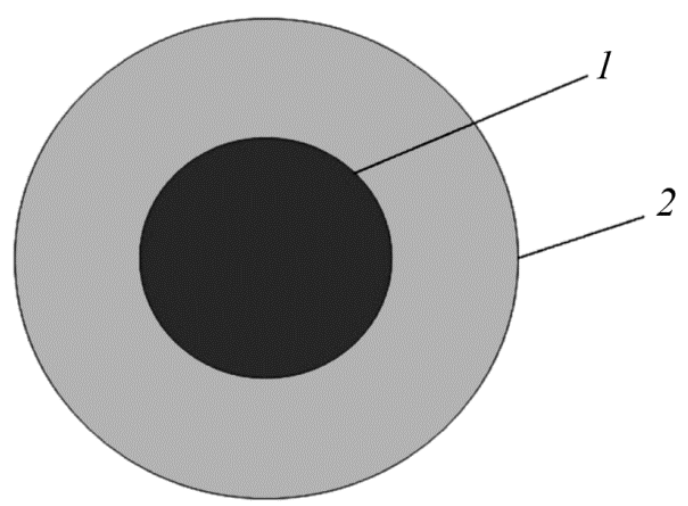

Рис. 1. Схематичное изображение комбинированного окна:

For citation: On the possibility of increasing the service life of high-power laser optics through the use of polycrystalline diamond windows with a central monocrystalline area / M. V. Rogozhin, V .E. Rogalin, M. I. Krymsky, I. A. Kaplunov // Diagnostics, Resource and Mechanics of materials and structures. - 2018. - Iss. 1. - P. 34-40. - DOI: 10.17804/24109908.2018.1.034-040. 
1 - монокристаллическая область; 2 - поликристаллическая область

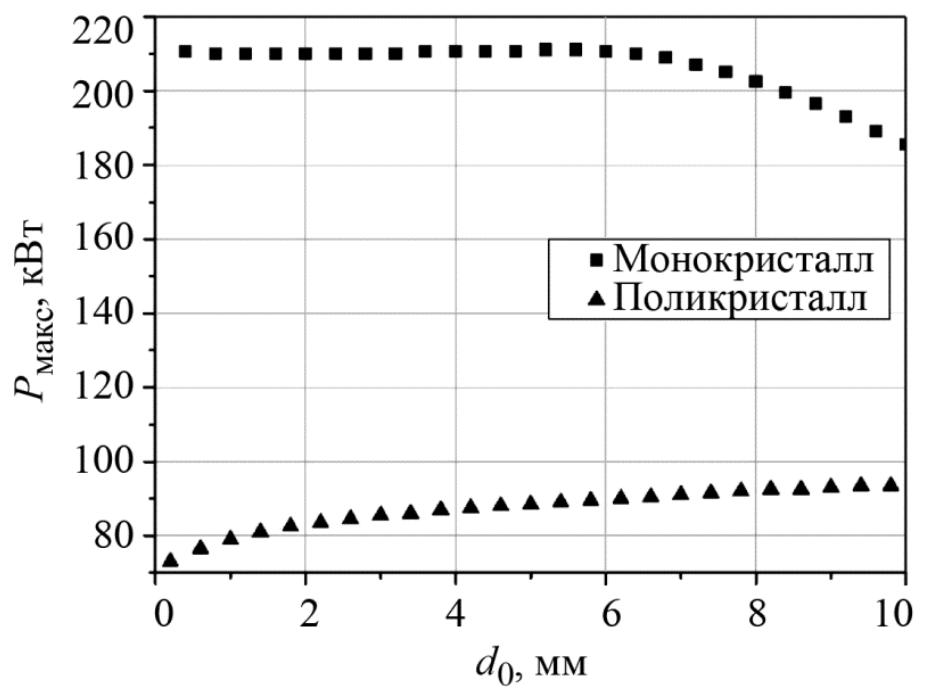

Рис. 2. Сравнение лучевой стойкости окон из поликристаллического и монокристалла алмаза

Проведен численный расчет, имеющий целью сравнение оптической стойкости окна из поликристаллического алмаза диаметром 20 мм и предложенного комбинированного окна аналогичного диаметра с центральной областью диаметром 5 мм, выполненной из монокристаллического алмаза (рис. 1). Для этого численно была смоделирована зависимость макси-

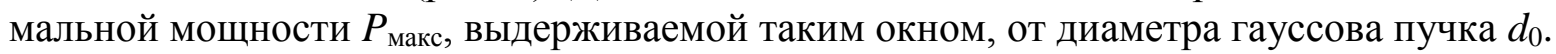

\section{3. Результаты и обсуждение}

На рис. 2 видно, что окно с центральной областью, выполненной из монокристаллического алмаза, разрушается при мощности порядка 210 кВт, причем максимальная мощность практически не зависит от размеров пучка. Падение максимальной мощности при $d_{0}>6$ мм (рис. 2) обусловлено выходом пятна лазерного излучения за пределы центральной области в поликристаллическую зону. При этом порог разрушения окна из поликристаллического алмаза аналогичного диаметра находится в диапазоне 80-100 кВт. Следует учесть, что в этих расчётах не учитываются другие возможные механизмы разрушения оптического элемента мощным лазерным излучением, в частности возможное разрушение алмаза при пробое в электрическом поле световой волны [1].

\section{4. Заключение}

Применение комбинированной алмазной пластины, рассмотренной выше конструкции позволяет повысить предел оптической стойкости выходного окна и, как следствие, максимальную мощность генерируемого излучения лазера не менее чем в 2 раза за счет увеличения предела механической прочности выходного окна и снижения количества поглощаемой в нем энергии вследствие снижения коэффициентов поглощения и рассеяния.

\section{Литература}

1. Рогалин В. Е. Прозрачные материалы для мощных импульсных $\mathrm{CO}_{2}$-лазеров // Известия ВУЗОВ. Материалы электронной техники. - 2013. - № 2. - С. 11-18.

2. Рогалин В. Е., Аранчий С. А. Поликристаллические алмазы - новые перспективы силовой оптики и электроники // Интеграл. - 2012. - № 5 (67). - С. 7-9. 
3. Resistance of diamond optics to high-power fiber laser radiation / V. E. Rogalin, E. E. Ashkenazi, A. F. Popovich, V. G. Ral'chenko, V. I. Konov, S. M. Aranchii, M. V. Ruzin, S. A. Uspenskii // Russian Microelectronics, 2012, vol. 41, no. 8, pp. 464-468. - DOI: 10.1134/S106373971208015X

4. High-order stimulated Raman scattering in CVD single crystal diamond / A. A. Kaminskii, R. J. Hemley, J. Lai, C. S. Yan, H. K. Mao, V. G. Ralchenko, H. J. Eichler, H. Rhee // Laser Phys. Lett. - 2007. - Vol. 4, iss. 5. - P. 350-353. - DOI: 10.1002/lapl.200610127

5. Development of high quality single crystal diamond for novel laser applications / I. Friel., S. L. Geoghegan, D. J. Twitchen, G. A. Scarsbrook // Proc. SPIE 7838, Optics and Photonics for Counterterrorism and Crime Fighting VI and Optical Materials in Defense Systems Technology VII. - 2010. - Vol. 783819.

6. Intrinsic and extrinsic absorption of chemical vapor deposition single-crystal diamond from the middle ultraviolet to the far infrared / S. Webster, Y. Chen, G. Turri, A. Bennett, B. Wickham, M. Bass // J. Opt. Soc. Am. B. - 2015. - Vol. 32, no. 3. - P. 479-484. - DOI: 10.1364/JOSAB.32.000479

7. Diamond optical components for high - power and high-energy laser applications / E. Anoikin, A. Muhr, An. Bennett, D. J. Twitchen, H. de Wit // Proc. of SPIE LASE, San Francisco, California, United States, 20 February 2015. - 2015. - Vol. 9346, 93460T. - P. 1-9. DOI: $10.1117 / 12.2079714$

8. Diode-pumped caesium vapour laser with closed-cycle laser-active medium circulation / A. V. Bogachev, S. G. Garanin, A. M. Dudov, V. A. Eroshenko, S. M. Kulikov, G. T. Mikaelyan, V. A. Panarin, V. O. Pautov, A. V. Rus, S. A. Sukharev. - Quantum Electronics. - 2012. - Vol. 42, no. 2. - P. 95-98. - DOI: 10.1070/QE2012v042n02ABEH014734

9. Prospects for large single crystal CVD diamond / S. Ho, C. S. Yan, Z. Liu, H. K. Mao, R. J. Hemley // Industrial Diamond Review. - 2006. - Vol. 66. - P. 28-32.

10. Fabrication of 1 Inch Mosaic Crystal Diamond Wafers / H. Yamada, A. Chayahara, Y. Mokuno, H. Umezawa, S. Shikata, N. Fujimori // Applied Physics Express. - 2010. - Vol. 3, no. 5. - P. 051301. - DOI: 10.1143/APEX.3.051301

11. Combined Single-crystalline and Polycrystalline CVD Diamond Substrates for Diamond Electronics / A. L. Vikharev, A. M. Gorbachev, M. P. Dukhnovsky, A. B. Muchnikov, A. K. Ratnikova, Yu. Yu. Fedorov // Semiconductors. - 2012. - Vol. 46, no. 2. - P. 263-266. DOI: $10.1134 / \mathrm{S} 1063782612020248$

12. Modeling the thermomechanical processes in the output window of a high-power $\mathrm{CO} 2$ laser / M. V. Rogozhin, M. I. Krimsky, V. E. Rogalin, S. A. Filin // Bulletin of the Russian Academy of Sciences: Physics. - 2016. - Vol. 80, no. 10. - P. 1260-1266. - DOI: 10.3103/S1062873816100166 13. Rogozhin M. V., Rogalin V. E. and Krymskii M. I. Thermooptical processes in the window of a high-power gas laser // Optics and Spectroscopy. - 2017. - Vol. 122. - No. 5. - P. 843-849. DOI: $10.1134 / \mathrm{S} 0030400 \mathrm{X} 17050186$

14. Мощный лазер : пат. 2608309 Рос. Федерация / Рогожин М. В., Рогалин В. Е., Крымский М. И., Филин С. А., Рогалина Н. А. - № 2015131054 : заявл. 27.07.2015 ; опубл. 17.01.2017, Бюл. № 2 . 